\title{
Thorium Isotopes, Particle Cycling Models, and Inverse Calculations of Model Rate Constants
}

\author{
Richard J. Murnane and Jorge L. SARmiento
}

\author{
Program in Atmospheric and Oceanic Sciences, Princeton University, Princeton, New Jersey
}

\author{
Michael P. Bacon
}

Department of Chemistry, Woods Hole Oceanographic Institution, Woods Hole, Massachusetts

\begin{abstract}
Generalized models of thorium and particle cycling, data from Station $\mathrm{P}$, and an inversion technique are used to obtain rate estimates of important biological and chemical transformations occurring in the water column. We first verify the inversion technique using an idealized data set generated by a finite difference model, and then apply the inversion technique to data from Station $P$. With the Station $P$ data, predicted rate constants for adsorption and release of thorium between the dissolved and small particle phases are consistent with the results from other workers. The predicted rate constants for the interaction between small and large particles are smaller than previous estimates. The predicted concentration of large rapidly sinking particles is greater than the concentration of suspended non-sinking particles, whereas the reverse is usually assumed to be the case. The calculated sinking rate for the large particles is $20 \mathrm{~m} \mathrm{~d}^{-1}$. This sinking rate is an order of magnitude smaller than the large particle sinking rate inferred from sediment trap mass fluxes at two levels in the water column. The reason we predict a high large particle concentration and slow settling velocity has not been uniquely determined. Possible modifications of the current model that could help to reconcile the differences between observations and model predictions include: 1) two classes of rapidly sinking particles or rate constants that change with depth, 2) direct interactions between the large particle and dissolved phases, and 3) incorporation of a continuous distribution of particle size and settling velocity.
\end{abstract}

\section{INTRODUCTION}

Particle cycling through the water column plays a central role in oceanic biogeochemistry. Sinking particles transfer substances from the surface ocean to the deep ocean and interact extensively with material that is suspended and dissolved in the water column. Our interests concern the particle interactions with nutrients and carbon. We want to use trace metals and radioactive isotopes to study these interactions. In this paper we describe and test an inverse technique that yields rate constants and error estimates for biogeochemical interactions.

Most of what we know about particle cycling in the oceans comes either indirectly from particle reactive trace metal radioisotope studies [e.g., Nozaki et al., 1981; Bacon and Anderson, 1982; Bacon et al., 1985; Coale and Bruland, 1985, 1987; Nozaki et al., 1987] or directly from sediment trap, large volume pumping, and/or other particle studies [e.g., Bishop et al., 1978; Honjo, 1980; Suess, 1980; Betzer et al., 1984; Martin et al., 1987; Walsh et al., 1988]. In this paper we use recent ideas regarding trace metal interactions with particles (focusing on thorium) as synthesized by Bacon et al. [1985], and further studied by Nozaki et al. [1987]. These authors proposed that particle-thorium interactions can be represented by a box model that involves: dissolved trace metals; adsorption of dissolved trace metals onto relatively abundant non-sinking fine particles; desorption

Copyright 1990 by the American Geophysical Union.

Paper number 90JC00753.

0148-0227/90/90JC-00753\$05.00 of trace metals from the fine particles; aggregation of fine particles into rapidly sinking large particles, which are considered to have a very low abundance in the water column; and disaggregation of large particles.

Using two western Pacific ${ }^{230} \mathrm{Th}$ profiles and the above box model Nozaki et al. [1987] succeeded in obtaining rate constants for adsorption and desorption of thorium and for particle aggregation and disaggregation. They assumed that the observed linear relation between thorium activity and depth extended to the surface. We show how to calculate estimates of the box model rate constants for any profile by solving simultaneous equations detailing the exchange of mass and thorium activity between the dissolved and various particulate phases. A parallel model for particle cycling includes processes that are usually thought to be biologically mediated and relates the trace metal cycling model directly to sediment trap and other particle measurements. We use a finite difference model based on the thorium model described above to generate a synthetic data set that is free of sampling and analytical errors. We then alter individual elements from the synthetic data set to test the sensitivity of rate constants predicted using the inverse technique. Finally, we apply the inverse technique to thorium data from Station $P$.

Results from the Station $P$ data are inconsistent with the basic assumptions underlying the development of the model and with field observations. In particular, we find that small particles are much lower in abundance than large particles, and we find that the large particles sink with a velocity that is almost an order of magnitude slower than inferred from sediment trap data. These inconsistencies suggest that the model assumptions are wrong. We offer some additional interactions that could be included in future models. 


\section{MODEL DESCRIPTION}

We use a trace metal cycling model (Figure 1a) analogous to that proposed by Bacon et al. [1985]. In addition to the rate constants for inorganic adsorption and desorption there is a rate constant associated with biologic processes that represents the active release of trace metals to solution. A simple particle model can incorporate the same phases and similar interactions (Figure 1b). The activity of a trace metal radioisotope is divided between the dissolved $\left(A_{d}\right)$, small particle $\left(A_{s}\right)$, and large particle $\left(A_{L}\right)$ phases. These definitions correspond to the operationally defined classes of dissolved material (material that passes through a filter), small particles (suspended material collected by filtration) and large particles (sinking material collected in sediment traps). Filtration collects large particles as well as small particles, but we assume that the amount of large particles collected by filtration is small enough to be ignored. $A$ is the activity in dpm $\mathrm{L}^{-1}$. We use the symbol $A^{\prime}$ to represent the activity per unit mass of particles $\left(\mathrm{dpm} \mathrm{g}^{-1}\right)$, i.e., $A_{L}=A_{L}^{\prime} P_{L} . P_{L}$ is the large particle concentration and $P_{s}$ is the small particle concentration, both in units of $\mathrm{g} \mathrm{L}^{-1}$. The quantities measured directly are $A_{d}, A_{s}, A_{L}^{\prime}$, and the large particle flux, $\omega P_{L}$. The large particle sinking velocity is $\omega$.

The small particle phase removes metal from solution by adsorption at a rate, $k_{1}$, that is dependent on metal chemistry. There appears to be a correlation between $k_{1}$ and a metal's hydrolysis energy [Li, 1981; Turner et al., 1981; Kumar, 1987; Clegg and Sarmiento, 1989]. The concentrations of surface adsorption sites and particles also affect the adsorption rate constant [Honeyman et al., 1988]. In addition, metals may be removed from the dissolved phase by active biologic uptake; however, this may be an insignificant reaction for strongly hydrolyzed metals and the actual extent of this process is poorly known. Collier and Edmond [1984] show that metal/phosphate ratios in biogenic particles can be relatively constant over a wide range of dissolved metal/phosphate ratios for "biointermediate" metals such as $\mathrm{Cu}$ and $\mathrm{Ni}$. In contrast, the observation that living and dead phytoplankton cells concentrate some transuranics to the same extent [Fisher et al., 1983; Fisher, 1986] implies that adsorption dominates the uptake by biota of strongly hydrolyzed trace metals. As a first approximation we assume that active biologic uptake of metals occurs only in the euphotic zone and we restrict our discussion to areas below the euphotic zone.

Metal is added to the dissolved phase through desorption from the small particles at a rate given by $k_{-1}$, and by biologically mediated remineralization of particles at a rate given by $\beta_{-1}$. $\beta_{2}$ and $\beta_{-2}$ are rate constants for the exchange of metal between the small and large particle phases as a result of particle aggregation and disaggregation. All rate constants are in units of $y^{-1}$. The flux of metal due to the sinking large particle phase is given by $F_{A_{L}}=\omega A_{L}^{\prime} P_{L}$. Finally, there is also loss of the metal from each of the phases by radioactive decay with a decay constant $\lambda y^{-1}$. The steady state conservation equation for the metal in each phase is

$$
0=S+A_{s}\left(k_{-1}+\beta_{-1}\right)-A_{d}\left(k_{1}+\lambda\right)+T_{A_{d}}
$$
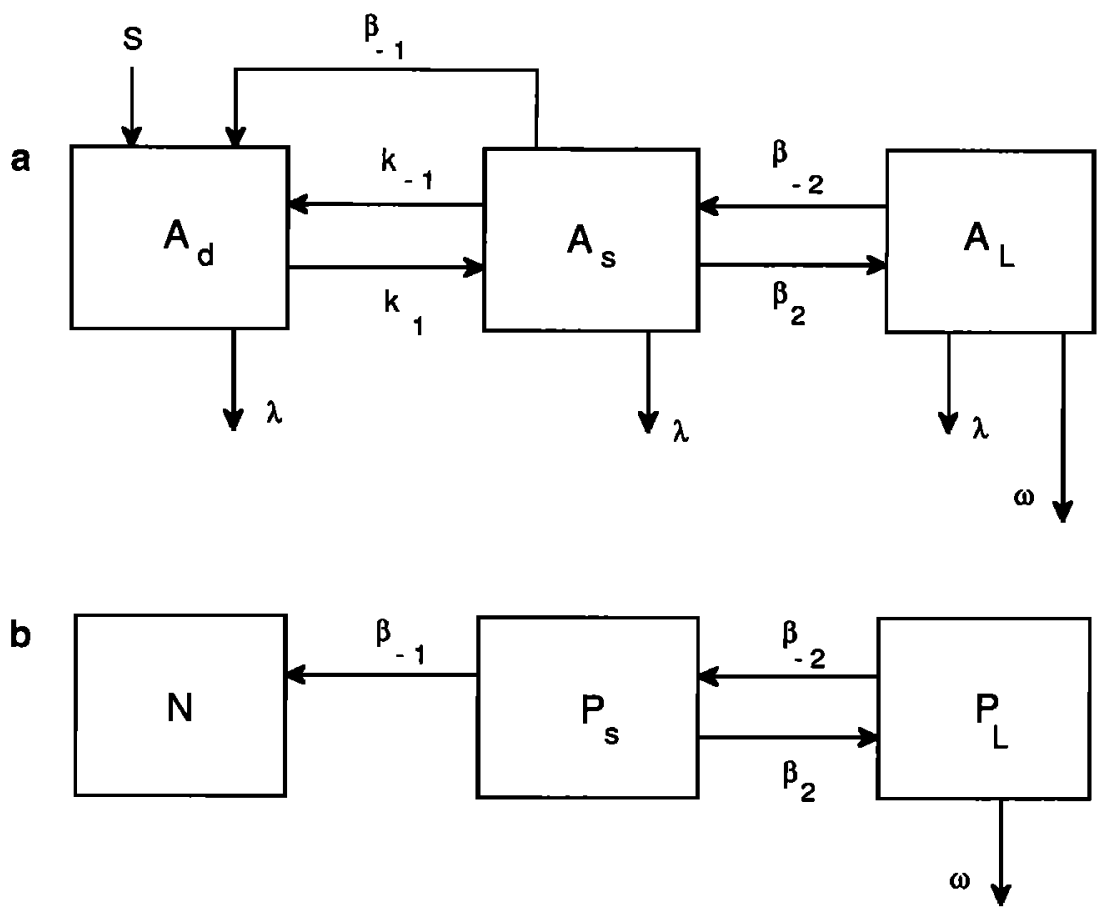

Fig. 1. Schematic diagrams of particle and trace metal cycling models below the euphotic zone. (a) Trace metal cycling. The $k$ 's denote adsorption and desorption reactions between the dissolved trace metal $\left(A_{d}\right)$ and trace metal in the small particle phase $\left(A_{s}\right)$. The trace metal associated with the large particle phase $\left(A_{L}\right)$ settles at a velocity, $\omega$. Radioactive decay is shown by $\lambda$, and $S$ denotes the production of daughter isotopes by a dissolved parent. The different $\beta$ 's are rate constants for the biologically mediated interactions that transfer metal between the different phases. (b) Particle cycling. Dissolved nutrients are denoted by $N$, small suspended particles by $P_{s}$, and large sinking particles by $P_{L}$. 


$$
\begin{aligned}
0= & A_{d} k_{1}+A_{L}^{\prime} P_{L} \beta_{-2}- \\
& A_{s}\left(k_{-1}+\beta_{-1}+\beta_{2}+\lambda\right)+T_{A_{s}} \\
0= & A_{s} \beta_{2}-A_{L}^{\prime} P_{L}\left(\beta_{-2}+\lambda\right)-\frac{\partial F_{A_{L}}}{\partial z}+T_{A_{L}}
\end{aligned}
$$

Equation (1a) describes the dissolved phase reactions, (1b) the small particle reactions, and (1c) the large particle reactions. $\mathrm{S}\left(\mathrm{dpm} \mathrm{L}^{-1} \mathrm{y}^{-1}\right)$, which arises from production of $A_{d}$ by decay of the parent, is the activity of the parent times the decay constant of the daughter. We use $A_{L}^{\prime} P_{L}$ rather than $A_{L}$ in the equations because $A_{L}^{\prime}$ is a measured quantity. $\mathrm{T}$, which represents the time derivative, advective, and diffusive contributions, is given by

$$
T_{()}=-\frac{\partial()}{\partial t}-\vec{V} \cdot \vec{\nabla}()+\vec{\nabla} \cdot K \vec{\nabla}()
$$

where $\vec{V}$ is velocity and $\mathbf{K}$ is a mixing coefficient that accounts for diffusive mixing processes. The equations describing particle cycling are analogous to Equation (1)

$$
\begin{aligned}
& 0=P_{s} R \beta_{-1}+T_{N} \\
& 0=P_{L} \beta_{-2}-P_{s}\left(\beta_{-1}+\beta_{2}\right)+T_{P_{s}} \\
& 0=P_{s} \beta_{2}-P_{L} \beta_{-2}-\omega \frac{\partial P_{L}}{\partial z}+T_{P_{L}}
\end{aligned}
$$

$R$ is a Redfield-like constant that gives the moles of nutrient per gram of particle. In what follows, we assume that $T_{P_{s}}, T_{P_{L}}, T_{A_{s}}$, and $T_{A_{L}}$ are negligible, in accord with the approach of Bacon and Anderson [1982] and Nozaki et al. [1987]. We will, however, consider the contributions of vertical mixing and small deviations from steady state to $T_{A_{d}}$ and $T_{N}$ when dealing with the finite difference model.

A consideration of Equation (1) shows that there are 3 equations and 7 unknowns: the $3 \beta$ 's, the two $k$ 's, $P_{L}$, and $T_{A_{d}}$. For our discussion, we take all the rate constants as first order. The number of unknowns can be reduced to 6 by combining $k_{-1}$ and $\beta_{-1}$ into a single term, $k_{-1}^{\prime}=$ $\left(k_{-1}+\beta_{-1}\right)$; and to 5 if we ignore $T_{A_{d}}$. This system of 3 equations with 5 or 6 unknowns can be solved at any depth in the water column by using data from three thorium isotopes (e.g., ${ }^{228} \mathrm{Th},{ }^{230} \mathrm{Th}$, and ${ }^{234} \mathrm{Th}$ ), which gives us up to 9 independent equations.

The flux gradient term for the large particles, $\partial F_{A_{L}} / \partial z$, and the large particle activity, $A_{L}^{\prime}$, present particular difficulties in solving the system of equations. The vertical resolution of $\partial F_{A_{L}} / \partial z$ and $A_{L}^{\prime}$ can be poor because sediment traps are generally spaced a considerable distance apart. Two approaches for obtaining these terms from sediment trap measurements are possible. In the first approach, one obtains an estimate for the flux gradient by taking the difference between the large particle flux at two traps and dividing by the separation between the traps

$$
\frac{\partial F}{\partial z} \approx \frac{\left(F_{t r a p_{2}}-F_{t r a p_{1}}\right)}{\Delta z}
$$

One then assumes that this estimate of the flux gradient applies to all depths between the traps where measurements of the dissolved and suspended particulates are made. In addition, some functional form for the variation of the large particle activity with depth between the traps must be assumed. With these assumptions, one then solves Equation (1) for the unknowns at each depth between the traps where measurements of $A_{d}$ and $A_{s}$ are obtained. The drawback of this approach is that data from sediment traps show clearly that the flux gradient changes with depth [e.g., Martin et al., 1987]. Furthermore, we lack good information for making an assumption on the functional form of the change in large particle activity with depth.

The alternate approach involves integrating the conservation equations between the two traps and dividing by the depth interval to obtain the average values for all terms in the equations. The flux gradient term remains the same: the difference in flux between the two traps divided by the depth interval. We assume that the large particle activity is the average of the activity in the two traps and use the depth averaged values for the dissolved and small particle activities. This method avoids problems with the particle concentration dependence of $k_{1}$ because we find the average value of $k_{1}$ for the given depth interval. The drawback of this approach is the problem of how to deal with the vertical integral of the product of two terms: an unknown rate constant (e.g., $\beta$ ) and an activity (e.g., $A$ ) measured at several depths between the traps. In order to solve for the average of $\beta, \bar{\beta}=\left(\int_{z_{1}}^{z_{2}} \beta d z\right) /\left(z_{2}-z_{1}\right)$, we must be able to assume that the integral of the product $\beta A$ is equal to the product of the integrals of $\beta$ and $A$, i.e.,

$$
\int_{z_{1}}^{z_{2}} \beta A d z \approx \int_{z_{1}}^{z_{2}} \beta d z \int_{z_{1}}^{z_{2}} A d z
$$

This is true only if $\beta$ and $A$ are uncorrelated, an unlikely condition. For example, the bacterial population is almost certainly a function of food supply, so that a term like $\beta_{-1}$ may be expected to change when the particle concentration varies. Despite these difficulties, we prefer this second approach to the first, and use it in what follows. Ultimately, better vertical resolution and more sophisticated models of the processes represented by the $\beta$ 's will remove or reduce these drawbacks. Such models should use parameters that may reasonably be assumed constant over large ocean regions. The type of modeling approach we envision is exemplified by the work of Toggweiler et al. [1987] and Sarmiento et al. [1989].

Matrix notation provides a convenient method of dealing with the equations describing the change in mass or activity of the different phases. By making all the assumptions described after Equation (1), and by grouping the unknown rate constants into a single vector, Equation (1) for each trace metal can be rewritten as

$$
\left(\begin{array}{cccccc}
-A_{d} & A_{s} & 0 & 0 & 0 & 1 \\
A_{d} & -A_{s} & -A_{s} & A_{L}^{\prime} & 0 & 0 \\
0 & 0 & A_{s} & -A_{L}^{\prime} & -A_{L}^{\prime} \lambda & 0
\end{array}\right)\left(\begin{array}{c}
k_{1} \\
k_{-1}^{\prime} \\
\beta_{2} \\
\beta_{-2} P_{L} \\
P_{L} \\
T_{A_{d}}
\end{array}\right)=
$$

$$
\left(\begin{array}{lll}
A_{d} \lambda-S & \left.A_{s} \lambda \quad \frac{\partial F_{A_{L}}}{\partial z}\right)^{\mathrm{T}}
\end{array}\right.
$$

Recall that $k_{-1}^{\prime}=\left(k_{-1}+\beta_{-1}\right)$ and note that $\mathbf{T}$ denotes the transpose of the vector.

Our task will be to solve for the unknowns in this set of equations. Solving these equations is straightforward if all the data are known. However, field data are subject to sampling error, analytical error, and possibly some systematic error; we try to account for such uncertainties in a solution. In addition, our assumption that the $T$ terms are negligible can be an over-simplification. A sensitivity 
analysis can be used to test for the effects of deviations from the model assumptions. We used a technique outlined by Wolberg [1967] that solves systems of linear and non-linear equations in a least squares sense assuming there is no data covariance. The technique can weight each term by its error and also yields a covariance matrix and confidence intervals for the solution. The method is very general and can easily be adapted to a variety of models as long as the problem is over-determined. With the linear system of Equations (3), $\beta_{-2}$ will have to be found from the calculated values of $\beta_{-2} P_{L}$ and $P_{L}$, thus the covariance between the errors in $\beta_{-2} P_{L}$ and $P_{L}$ must be considered.

We next discuss the development of a finite difference model which includes mixing of the dissolved component. The data are used to test our inverse modeling technique, and the sensitivity of the predicted solution is explored by varying the output of the finite difference model.

\section{Finite Difference Model}

A full mathematical description of oceanic conditions requires advective and diffusive terms in three dimensions as well as information on any additional source and sink terms. We are presently working on such models, but here we restrict our modeling efforts and discussion to the onedimensional steady state case. The finite difference model includes sinking of large particles and diffusive mixing of dissolved thorium. Rate constants for metal cycling are taken from Nozaki et al. [1987] and assumed to be constant. $k_{1}$ is dependent on particle concentration and surface area [Honeyman et al., 1988]; however, the calculated results based on a constant value for $k_{1}$ are adequate for testing the inverse modeling scheme.

The $4000 \mathrm{~m}$ deep model ocean is divided into 27 layers of varying thickness. Each of the top three layers is 10 $m$ thick. Large gradients in the surface ocean require a more closely spaced grid for numeric stability. The layers gradually thicken to the point where the bottom four layers are each $400 \mathrm{~m}$ thick. The large particle class sinks at a rate of $100 \mathrm{~m} \mathrm{~d}^{-1}$. The small particles do not sink.

Three thorium isotopes were included in the model: ${ }^{234} \mathrm{Th},{ }^{230} \mathrm{Th}$, and ${ }^{228} \mathrm{Th}$. The source terms for ${ }^{234} \mathrm{Th}$ and ${ }^{230} \mathrm{Th}$ are easily stated and well known. The parents of ${ }^{234} \mathrm{Th}$ and ${ }^{230} \mathrm{Th}\left({ }^{238} \mathrm{U}\right.$ and $\left.{ }^{234} \mathrm{U}\right)$ are homogeneously distributed through the ocean with known activities. The distribution of ${ }^{228} \mathrm{Ra}$, the parent of ${ }^{228} \mathrm{Th}$, is less constrained. We calculated a steady state ${ }^{228} \mathrm{Ra}$ profile (Figure 2) using an eddy diffusion coefficient of $1 \mathrm{~cm}^{2}$ $\sec ^{-1}$ and assumed that there was a ${ }^{228} \mathrm{Ra}$ surface flux of 1 atom $\mathrm{cm}^{-2} \mathrm{~min}^{-1}$ and a bottom flux of 0.5 atom $\mathrm{cm}^{-2}$ $\min ^{-1}$. These fluxes are fairly typical for the Pacific Ocean [Sarmiento, 1978; Cochran, 1979].

The following equations were solved at each depth level in the model:

$$
\begin{aligned}
& \frac{\partial A_{d}}{\partial t}=S+A_{s} k_{-1}^{\prime}-A_{d}\left(k_{1}+\lambda\right)+K_{v} \frac{\partial^{2} A_{d}}{\partial z^{2}} \\
& \frac{\partial A_{s}}{\partial t}=A_{d} k_{1}+A_{L} \beta_{-2}-A_{s}\left(k_{-1}^{\prime}+\beta_{2}+\lambda\right) \\
& \frac{\partial A_{L}}{\partial t}=A_{s} \beta_{2}-A_{L}\left(\beta_{-2}+\lambda\right)-\omega \frac{\partial A_{L}}{\partial z}
\end{aligned}
$$

where $K_{v}$, the vertical eddy diffusion coefficient, and $\partial / \partial t$ are the only terms in $T_{()}$that we preserve. These equations differ from Equation (1) in that we use $A_{L}$ rather than $A_{L}^{\prime} P_{L}$ and we use $k_{-1}^{\prime}$ instead of $k_{-1}$ and $\beta_{-1}$. This saves us the necessity of solving the additional particle conservation equations that would arise from the model in Figure 1b. The initial conditions of the model had a constant activity for the dissolved thorium isotopes through the water column and no activity in the two particulate phases (Table 1). We used a short time step of 33.3 minutes because of the fast settling velocity of the large particles and the thinness of the model's top levels $(10 \mathrm{~m})$. Thorium adsorbed onto the large particles was removed from the ocean as it sank out of the bottom layer. The model was run out for 1000 years.

TABLE 1. Constants and Initial Conditions for the Finite Difference Model

\begin{tabular}{ll}
\hline Model Constants & \multicolumn{1}{c}{ Initial Conditions } \\
\hline$k_{1}=0.195 \mathrm{y}^{-1}$ & ${ }^{238} \mathrm{U}=2.4 \mathrm{dpm} \mathrm{L} \mathrm{L}^{-1}$ \\
$k_{-1}=0.879 \mathrm{y}^{-1}$ & ${ }^{234} \mathrm{U}=2.76 \mathrm{dpm} \mathrm{L} \mathrm{L}^{-1}$ \\
$\beta_{2}=12.25 \mathrm{y}^{-1}$ & dissolved ${ }^{234} \mathrm{Th}=2.4 \mathrm{dpm} \mathrm{L} \mathrm{L}^{-1}$ \\
$\beta_{-2}=785 \mathrm{y}^{-1}$ & dissolved ${ }^{230} \mathrm{Th}=1 \times 10^{-3} \mathrm{dpm} \mathrm{L}^{-1}$ \\
$\omega=100 \mathrm{~m} \mathrm{~d}^{-1}$ & dissolved ${ }^{228} \mathrm{Th}=1 \times 10^{-3} \mathrm{dpm} \mathrm{L}^{-1}$ \\
$K_{v}=1 \mathrm{~cm}^{2} \mathrm{~s}^{-1}$ & small particle activity $=0 \mathrm{dpm} \mathrm{L} \mathrm{L}^{-1}$ \\
& large particle activity $=0 \mathrm{dpm} \mathrm{\textrm {L } ^ { - 1 }}$ \\
& $228 \mathrm{Ra}=0.061 \exp \left(-6.17 \times 10^{-5} z\right)+$ \\
& $0.0308 \exp \left(-6.17 \times 10^{-5}\left(4 \times 10^{5}-z\right)\right)$ \\
\hline
\end{tabular}

Rate constants are taken from Nozaki et al. [1987]

The predicted activities of the three thorium isotopes in the different phases are in reasonable agreement with data from oceanic profiles (Figure 2). The maximums in dissolved ${ }^{228} \mathrm{Th}$ at the surface and bottom are caused by the distribution of ${ }^{228} \mathrm{Ra}$. In contrast, the small and large particle ${ }^{228} \mathrm{Th}$ phases have sub-surface maxima that are produced by the kinetics of $\mathrm{Th}$ adsorption and particle settling. The ${ }^{230} \mathrm{Th}$ activity for all three phases increases with depth. The dissolved and small particle activities are close to those observed by Nozaki et al. [1987] over the same depth range. This suggests that a constant $k_{1}$ is adequate for modeling deep ocean ${ }^{230} \mathrm{Th}$. Dissolved ${ }^{234} \mathrm{Th}$ has a constant activity throughout the water column. The small and large particle phases have low ${ }^{234} \mathrm{Th}$ activities near the surface that increase with depth to almost constant values. The shapes of these profiles reflect the one-dimensional steady state nature of the model. Nonsteady state conditions, lateral advection, or changing rate constants would produce deviations from these profiles, such as the mid-water maximum in ${ }^{230} \mathrm{Th}$ observed by Bacon and Anderson [1982].

The activities predicted by the finite difference model were used as an idealized data set to test the inversion technique. The activity data for the three phases and three isotopes provided nine equations. The rate constants calculated with the inversion technique match those used in the finite difference model only when the contribution from $\partial A / \partial t$ is included (Table 2). Even a small change in activity for a time step relative to the activity of a given phase $\left(\Delta t(\partial A / \partial t) / A<10^{-5}\right.$, where $\Delta t$ is the time step) can alter the rate constants predicted with the inverse technique. The rate constants predicted with the inverse technique are insensitive to small changes in the diffusive mixing term; omitting the diffusion term from the inversion does not alter the predicted rate constants (Table 2). 
228Ra Activity

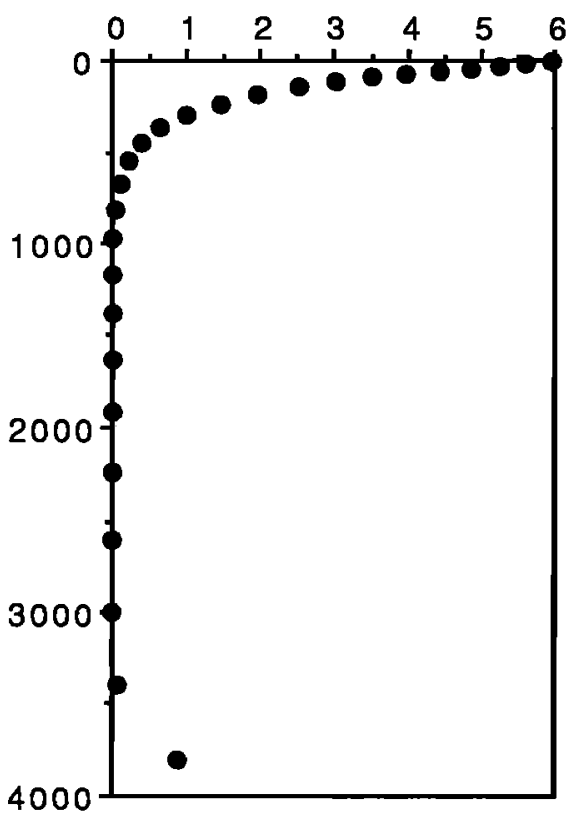

Depth $(\mathrm{m})$

a

228Th Activity

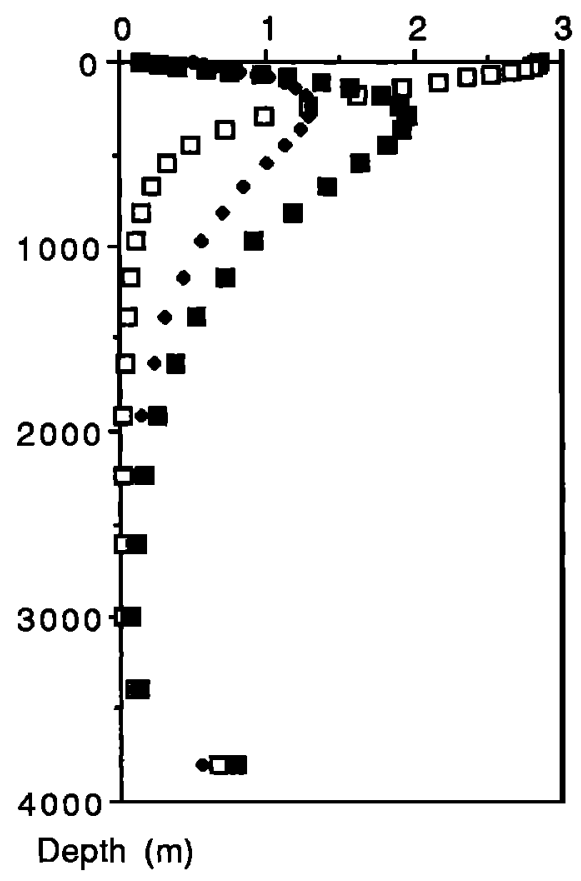

C
234Th Activity

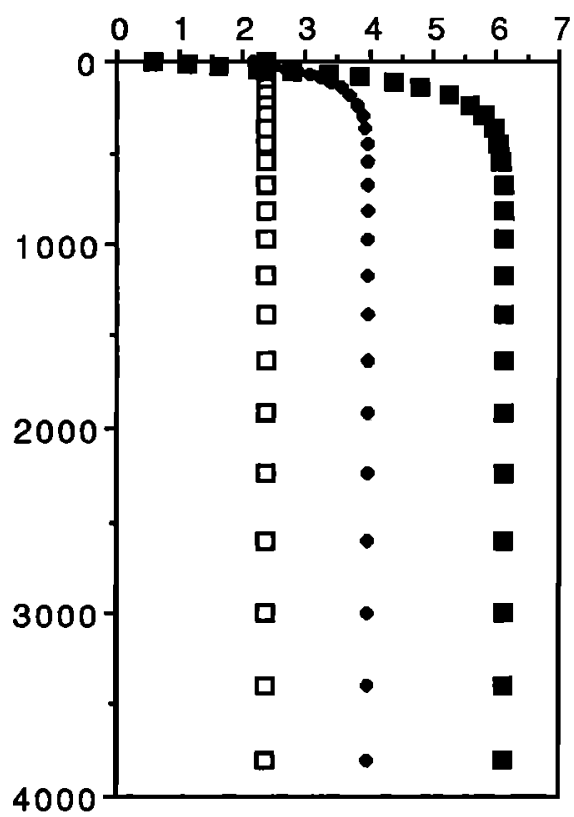

Depth (m)

b

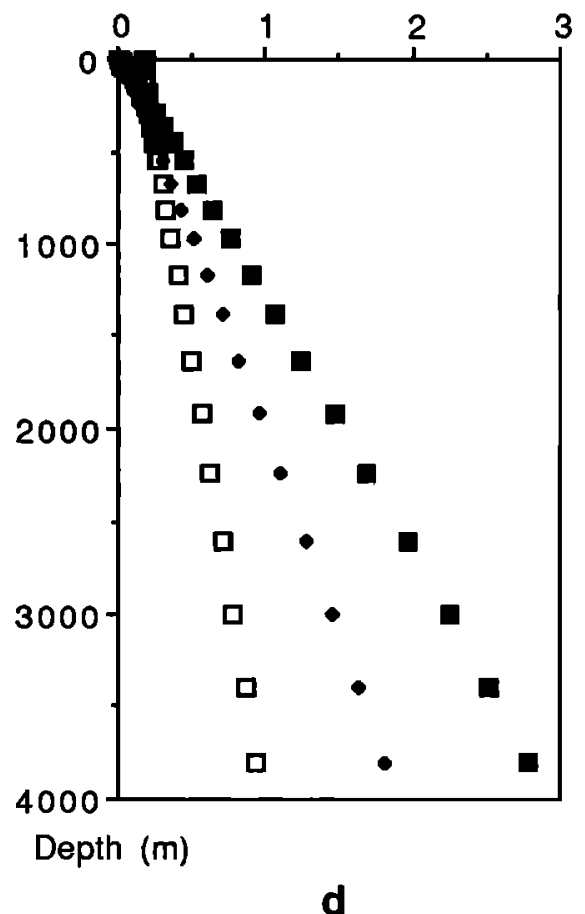

Fig. 2. Activity versus depth profiles for ${ }^{228} \mathrm{Ra},{ }^{234} \mathrm{Th},{ }^{230} \mathrm{Th}$, and ${ }^{228} \mathrm{Th}$ obtained from the one-dimensional diffusion model. (a) Activity values for ${ }^{228} \mathrm{Ra}$ (dpm $100 \mathrm{~L}^{-1}$ ) used in finite difference model. (b) ${ }^{234} \mathrm{Th}$ activity in the dissolved (open squares, $\mathrm{dpm} \mathrm{L}^{-1}$ ), small particle (diamonds, $\mathrm{dpm} 100 \mathrm{~L}^{-1}$ ), and large particle (filled squares, dpm $10^{4} \mathrm{~L}^{-1}$ ) phases. (c) ${ }^{228} \mathrm{Th}$ activity in the dissolved (open squares, dpm 100L ${ }^{-1}$ ), small particle (diamonds, dpm $10^{3} \mathrm{~L}^{-1}$ ), and large particle (filled squares, dpm $10^{5} \mathrm{~L}^{-1}$ ) phases. (d) ${ }^{230} \mathrm{Th}$ activity in the dissolved (open squares, dpm $10^{3} \mathrm{~L}^{-1}$ ), small particle (diamonds, dpm $10^{4} \mathrm{~L}^{-1}$ ), and large particle (filled squares, dpm $10^{6} \mathrm{~L}^{-1}$ ) phases. 
TABLE 2. Rate Constants $\left(y^{-1}\right)$ Predicted With Inverse Technique Using Finite Difference Model Data

\begin{tabular}{lcccc}
\hline \multicolumn{1}{c}{ Source } & $k_{1}$ & $k_{-1}$ & $\beta_{2}$ & $\beta_{-2}$ \\
\hline Nozaki et al. [1987] & $\mathbf{0 . 1 9 5}$ & $\mathbf{0 . 8 7 9}$ & 12.25 & 785 \\
Inversion including diffusion and $\partial A / \partial t$ & $\mathbf{0 . 1 9 5 \pm 0 . 0 0 0}$ & $\mathbf{0 . 8 7 9 \pm 0 . 0 0 0}$ & $\mathbf{1 2 . 2 5} \pm 0.01$ & $785 \pm 1$ \\
Inversion without diffusion & $\mathbf{0 . 1 9 5 \pm 0 . 0 0 0}$ & $\mathbf{0 . 8 7 9 \pm 0 . 0 0 0}$ & $\mathbf{1 2 . 2 5} \pm 0.01$ & $785 \pm 1$ \\
Inversion without $\partial A / \partial t$ & $0.205 \pm 0.014$ & $\mathbf{1 . 0 8 4 \pm 0 . 0 8 7}$ & $14 \pm 16$ & $890 \pm 1000$ \\
\hline
\end{tabular}

Errors are one standard deviation. Rate constants calculated from data at $3000 \mathrm{~m}$ in finite difference model.

We used the inversion technique with data from a variety of depths and tested the sensitivity of the predicted rate constants by systematically changing the activity of each isotope in each phase (Table 3). For our discussion we focus on data from the $3000 \mathrm{~m}$ level. The thorium activity predicted by the finite difference model for each phase was altered by $10 \%$ because this approximates the analytical error in the Th analyses. The sensitivity tests were carried out using nine equations for the three isotopes and included terms for diffusion and $\partial A / \partial t$.

The sensitivity of the rate constants predicted with the inverse technique is quite variable. $k_{1}$ and $k_{-1}$ are most sensitive to changes in the ${ }^{234} \mathrm{Th}$ dissolved phase activity. The source of this sensitivity is probably related to the fact that the ${ }^{234} \mathrm{Th}$ decay constant is larger than either rate constant. Changing the dissolved ${ }^{228} \mathrm{Th}$ activity has an intermediate effect on the predicted rate constants, while changing the dissolved ${ }^{230}$ Th has the smallest effect. $\beta_{2}$ and $\beta_{-2}$ are sensitive to changes in any phase except dissolved ${ }^{230} \mathrm{Th} . \beta_{2}$ and $\beta_{-2}$ are relatively insensitive to changes in the term $\omega \partial A_{L} / \partial z$. We do not completely understand why this is so. In the ${ }^{234} \mathrm{Th}$ equation, $\omega \partial A_{L} / \partial z$ and any changes to the term are insignificant when added to $A_{L} \lambda$, and there is no change in the rate constants. For the ${ }^{230} \mathrm{Th}$ equation, one can neglect $A_{L} \lambda$, and changing $\omega \partial A_{L} / \partial z$ has a relatively small affect on $\beta_{2}$ and $\beta_{-2}$ compared to changing $A_{s}$ or $A_{L}$. The ${ }^{228} \mathrm{Th}$ equation falls between these two extremes, but of the three isotopes, changing its activity flux gradient has the largest affect on the rate constants. A dimensional analysis of the equations suggests that the flux term is relatively small and that the change in the rate constants is related to the size of the flux term compared to the term $\left(A_{s} \beta_{2}+A_{L} \beta_{-2}\right)$.

Changes in the small and large particle activity strongly affect $\beta_{2}$ and $\beta_{-2}$ because $\beta_{2}$ and $\beta_{-2}$ are greater than the decay constants for all the isotopes. The fast interaction between the small and large particles causes the ratio of small to large particle activities for each isotope to be nearly equal. As a result, one column of the matrix in Equation (3) differs from another column by a nearly constant factor, in other words, two columns are almost collinear. The rate constants associated with the collinear columns, $\beta_{2}$ and $\beta_{-2}$, are "poorly resolved" by the activity data. Small changes in these columns (the small or large particle activities) can strongly affect solutions for $\beta_{2}$ and $\beta_{-2}$.

\section{Station P}

\section{Data and Results}

In order to calculate the rate constants and large particle concentration in Equation (3), one needs enough thorium isotope data to solve for the five unknowns $\left(T_{A_{d}}\right.$ is assumed to be 0, as in Bacon and Anderson [1982]; and Nozaki et al. [1987]). A suitable ${ }^{234} \mathrm{Th},{ }^{230} \mathrm{Th}$, and ${ }^{228} \mathrm{Th}$ data set was

TABLE 3. Rate Constant Sensitivity

\begin{tabular}{|c|c|c|c|c|c|c|c|c|}
\hline & \multicolumn{4}{|c|}{$\begin{array}{l}\text { Normalized Rate Constant After } \\
10 \% \text { Increase In Altered Term }\end{array}$} & \multicolumn{4}{|c|}{$\begin{array}{l}\text { Normalized Rate Constant After } \\
10 \% \text { Decrease In Altered Term }\end{array}$} \\
\hline & $k_{1}$ & $k_{-1}$ & $\beta_{2}$ & $\beta_{-2}$ & $k_{1}$ & $k_{-1}$ & $\beta_{2}$ & $\beta_{-2}$ \\
\hline Unaltered Value & 0.195 & 0.879 & 12.25 & 785 & 0.195 & 0.879 & 12.25 & 785 \\
\hline $\begin{array}{l}\text { Altered Term } \\
\text { Dissolved }{ }^{234} \mathrm{Th} \\
\text { Dissolved }{ }^{230} \mathrm{Th} \\
\text { Dissolved }{ }^{228} \mathrm{Th}\end{array}$ & $\begin{array}{l}0.631 \\
0.979 \\
1.056\end{array}$ & $\begin{array}{l}0.673 \\
1.025 \\
1.245\end{array}$ & $\begin{array}{l}0.41 \\
0.99 \\
1.10\end{array}$ & $\begin{array}{l}0.44 \\
0.99 \\
1.10\end{array}$ & $\begin{array}{l}6.031 \\
1.015 \\
0.949\end{array}$ & $\begin{array}{l}5.298 \\
0.965 \\
0.836\end{array}$ & $\begin{array}{l}1.63 \\
0.99 \\
0.92\end{array}$ & $\begin{array}{l}1.56 \\
0.99 \\
0.92\end{array}$ \\
\hline $\begin{array}{l}\text { Small Particle }{ }^{234} \mathrm{Th} \\
\text { Small Particle }{ }^{230} \mathrm{Th} \\
\text { Small Particle }{ }^{228} \mathrm{Th}\end{array}$ & $\begin{array}{l}1.051 \\
0.995 \\
0.974\end{array}$ & $\begin{array}{l}1.047 \\
0.954 \\
0.919\end{array}$ & $\begin{array}{r}0.38 \\
0.35 \\
-2.06\end{array}$ & $\begin{array}{r}0.39 \\
0.37 \\
-2.10\end{array}$ & $\begin{array}{l}0.918 \\
0.769 \\
1.015\end{array}$ & $\begin{array}{l}0.919 \\
0.562 \\
1.083\end{array}$ & $\begin{array}{l}0.23 \\
0.01 \\
0.38\end{array}$ & $\begin{array}{r}0.23 \\
-0.00 \\
0.37\end{array}$ \\
\hline $\begin{array}{l}\text { Large Particle }{ }^{234} \mathrm{Th} \\
\text { Large Particle }{ }^{230} \mathrm{Th} \\
\text { Large Particle }{ }^{228} \mathrm{Th}\end{array}$ & $\begin{array}{l}0.974 \\
0.933 \\
1.000\end{array}$ & $\begin{array}{l}0.973 \\
0.890 \\
1.000\end{array}$ & $\begin{array}{l}0.33 \\
0.07 \\
0.40\end{array}$ & $\begin{array}{l}0.33 \\
0.08 \\
0.39\end{array}$ & $\begin{array}{l}1.005 \\
0.985 \\
1.000\end{array}$ & $\begin{array}{l}1.007 \\
0.983 \\
1.000\end{array}$ & $\begin{array}{r}0.38 \\
0.35 \\
-1.70\end{array}$ & $\begin{array}{r}0.40 \\
0.37 \\
-1.74\end{array}$ \\
\hline $\begin{array}{l}{ }^{234} \mathrm{Th}: \omega \partial A_{L} / \partial z \\
{ }^{230} \mathrm{Th}: \omega \partial A_{L} / \partial z \\
{ }^{228} \mathrm{Th}: \omega \partial A_{L} / \partial z\end{array}$ & $\begin{array}{l}1.000 \\
1.000 \\
1.000\end{array}$ & $\begin{array}{l}1.000 \\
1.000 \\
1.000\end{array}$ & $\begin{array}{l}1.00 \\
1.01 \\
1.04\end{array}$ & $\begin{array}{l}1.00 \\
1.01 \\
1.04\end{array}$ & $\begin{array}{l}1.000 \\
1.000 \\
1.000\end{array}$ & $\begin{array}{l}1.000 \\
1.000 \\
1.000\end{array}$ & $\begin{array}{l}1.00 \\
0.99 \\
0.96\end{array}$ & $\begin{array}{l}1.00 \\
0.99 \\
0.96\end{array}$ \\
\hline
\end{tabular}

Rate constants predicted by inverse technique with finite difference model data from $3000 \mathrm{~m}$. The three equations for each thorium isotope were used in the inversion. Rate constants are normalized with respect to their unaltered value. 
collected at Station P. Dissolved and suspended sediment samples were collected at five depths between $1000 \mathrm{~m}$ and $3600 \mathrm{~m}$ (Table 4). Sediment trap data at $1000 \mathrm{~m}$ and $3800 \mathrm{~m}$ were collected at two week intervals from 27 March 1983 to 6 October 1983. The mass flux at each depth was quite variable during this period (Figure 3a). This highlights the importance of obtaining a sufficiently long time series of measurements so that one can obtain an average that satisfies the steady state assumptions of the model. This data set is marginal in this respect. A full description of the experimental data and an analysis will be given in a separate paper.

We transformed the field data into a structure suitable for the regression technique in the following manner. The average large particle activity at each sediment trap $\left(\bar{A}_{L}^{\prime}\right.$ dpm $\mathrm{g}^{-1}$ ) was calculated from the time weighted average mass flux $\left(\left(\sum F_{P_{L}} \Delta t\right) /\left(\sum \Delta t\right)\right)$ and the time weighted average large particle activity flux $\left(\left(\sum F_{P_{L}} A_{L}^{\prime} \Delta t\right) /\left(\sum \Delta t\right)\right)$. The large particle activity used in the inverse calculation is the average $A_{L}^{\prime}$ of the two depths. $\omega \partial A_{L} / \partial z$ is found by dividing the difference in the average activity fluxes by the depth difference between the traps. Finally, we use the ${ }^{234} \mathrm{Th},{ }^{230} \mathrm{Th}$, and ${ }^{228} \mathrm{Th}$ dissolved and suspended phase depth averaged activities between the sediment traps (Table 4).

Once the field data is properly averaged, the inverse technique can be used to find values for the rate constants and the large particle concentration $\left(k_{1}, k_{-1}, \beta_{2}, \beta_{-2} P_{L}\right.$, $P_{L}$, Table 5). We assume each activity and $\omega \partial A_{L} / \partial z$ have an error of $\pm 10 \%$, except for ${ }^{234} \mathrm{U}$ and ${ }^{238} \mathrm{U}$. Predicted values of $k_{1}\left(0.47 \mathrm{y}^{-1}\right)$ and $k_{-1}\left(0.97 \mathrm{y}^{-1}\right)$ are in reasonable agreement with previous estimates based on oceanographic data [Bacon and Anderson, 1982; Nozaki et al., 1987]. The sensitivity study of the finite difference model suggests that $k_{1}$ and $k_{-1}$ are the parts of the solution least affected by deviations from steady state and 1-D assumptions. The predicted concentration of large particles $\left(P_{L}\right)$ is $12 \mu \mathrm{g} \mathrm{L}^{-1}$ and seems rather high on first inspection. It resembles a value that might approximate the total suspended matter concentration. $\beta_{-2}$, which is calculated from $P_{L}$ and $\beta_{-2} P_{L}$, has a value of $0.8 \mathrm{y}^{-1}$, and $\beta_{2}$ has a value of $0.22 \mathrm{y}^{-1}$. The values of $\beta_{2}$ and $\beta_{-2}$ are smaller than those predicted by Nozaki et al. $\left[1987 ; \beta_{2}=2.4\right.$ to $12.3 \mathrm{y}^{-1}$ and $\beta_{-2}=148$ to $789 \mathrm{y}^{-1}$, for a settling velocity of $100 \mathrm{~m} \mathrm{~d}^{-1}$, or 350 to $2.4 \times 10^{4} \mathrm{y}^{-1}$, for a settling velocity of $300 \mathrm{~m} \mathrm{~d}^{-1}$ ).

\section{Discussion}

There is a dramatic difference between the values of $\beta_{2}$ and $\beta_{-2}$ based on the Station P data and the values of $\beta_{2}$ and $\beta_{-2}$ from Nozaki et al. [1987]. One would expect some difference because the ${ }^{230} \mathrm{Th}$ activity profiles are different. Still, such a large difference in rate constants requires some comment. The beta rate constants found by Nozaki et al. [1987] are greater than the ${ }^{228} \mathrm{Th}$ decay constant and on the order of, or greater than, the rate large particles travel through the water column. If $\beta_{2}$ and $\beta_{-2}$ were as great or greater than those found by Nozaki et al. [1987], then the

TABLE 4a. Station PAPA Data (Large Volume Data)

\begin{tabular}{lccccccc}
\hline & \multicolumn{3}{c}{ Particulate } & & \multicolumn{3}{c}{ Dissolved } \\
\cline { 2 - 4 } \cline { 7 - 8 } Depth, m & ${ }^{234} \mathrm{Th} \frac{\mathrm{dpm}}{\mathrm{L}}$ & ${ }^{230} \mathrm{Th} \frac{\mathrm{dpm}}{10^{3} \mathrm{~L}}$ & ${ }^{228} \mathrm{Th} \frac{\mathrm{dpm}}{10^{3} \mathrm{~L}}$ & & ${ }^{234} \mathrm{Th} \frac{\mathrm{dpm}}{\mathrm{L}}$ & ${ }^{230} \mathrm{Th} \frac{\mathrm{dpm}}{10^{3} \mathrm{~L}}$ & ${ }^{228} \mathrm{Th} \frac{\mathrm{dpm}}{10^{3} \mathrm{~L}}$ \\
\hline 1000 & 0.417 & 0.085 & 0.250 & & 2.08 & 0.187 & 0.569 \\
1800 & 0.279 & 0.103 & 0.191 & & 2.36 & 0.224 & 0.143 \\
2200 & 0.211 & 0.154 & 0.136 & & 2.50 & 0.402 & 0.249 \\
3100 & 0.165 & 0.206 & 0.155 & & 2.28 & 0.419 & 0.502 \\
3600 & 0.118 & 0.171 & 0.199 & & 2.26 & 0.541 & 1.130 \\
Average & 0.229 & 0.149 & 0.179 & & 2.32 & 0.359 & 0.474 \\
\hline
\end{tabular}

TABLE 4b. Station P Data (Sediment Trap Data)

\begin{tabular}{|c|c|c|c|c|c|c|}
\hline \multirow{2}{*}{$\begin{array}{l}\text { Sample } \\
\text { Interval }\end{array}$} & \multicolumn{3}{|c|}{$1000 \mathrm{~m}$} & \multicolumn{3}{|c|}{$3800 \mathrm{~m}$} \\
\hline & Mass Flux $\frac{m g}{m^{2} d}$ & ${ }^{230} \mathrm{Th} \frac{\mathrm{dpm}}{\mathrm{g}}$ & ${ }^{228} \mathrm{Th} \frac{\text { dpm }}{\mathrm{g}}$ & Mass Flux $\frac{\mathrm{mg}}{\mathrm{m}^{2} \mathrm{~d}}$ & ${ }^{230} \mathrm{Th} \frac{\mathrm{dpm}}{\mathrm{g}}$ & ${ }^{228} \mathrm{Th} \frac{\mathrm{dpm}}{\mathrm{g}}$ \\
\hline 1 & 176 & 0.32 & 6.80 & 94.7 & 1.98 & 7.49 \\
\hline 2 & 199 & 0.34 & 7.50 & 71.8 & 2.51 & 7.66 \\
\hline 3 & 323 & 0.28 & 5.76 & 121.8 & 1.93 & 5.93 \\
\hline 4 & 443 & 0.21 & 4.45 & 215.1 & 1.37 & 5.39 \\
\hline 5 & 295 & 0.28 & 4.64 & 221.0 & 1.39 & 5.30 \\
\hline 6 & 158 & 0.48 & 6.70 & 129.5 & 2.15 & 6.24 \\
\hline 7 & 420 & 0.23 & 3.33 & 99.1 & 2.63 & 6.76 \\
\hline 8 & 596 & 0.21 & 3.62 & 466.2 & 0.94 & 3.22 \\
\hline 9 & 348 & 1.09 & 4.42 & 427.7 & 1.25 & 5.35 \\
\hline 10 & 143 & 0.33 & 8.38 & 363.3 & 0.92 & 4.67 \\
\hline 11 & 93 & 0.70 & 11.64 & 135.2 & 0.69 & 12.16 \\
\hline 12 & 119 & 0.57 & 8.64 & 65.0 & 3.75 & 12.18 \\
\hline average & 276 & 0.38 & 5.24 & 201 & 1.40 & 5.69 \\
\hline
\end{tabular}

All sample intervals were 14 days. Average values were calculated using the methods noted in the text. 


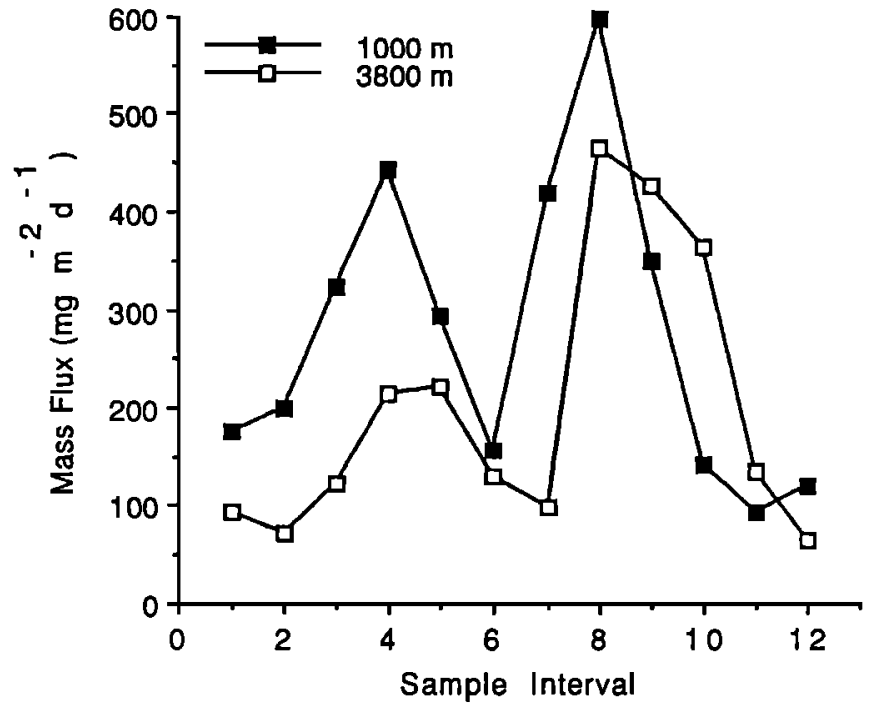

a

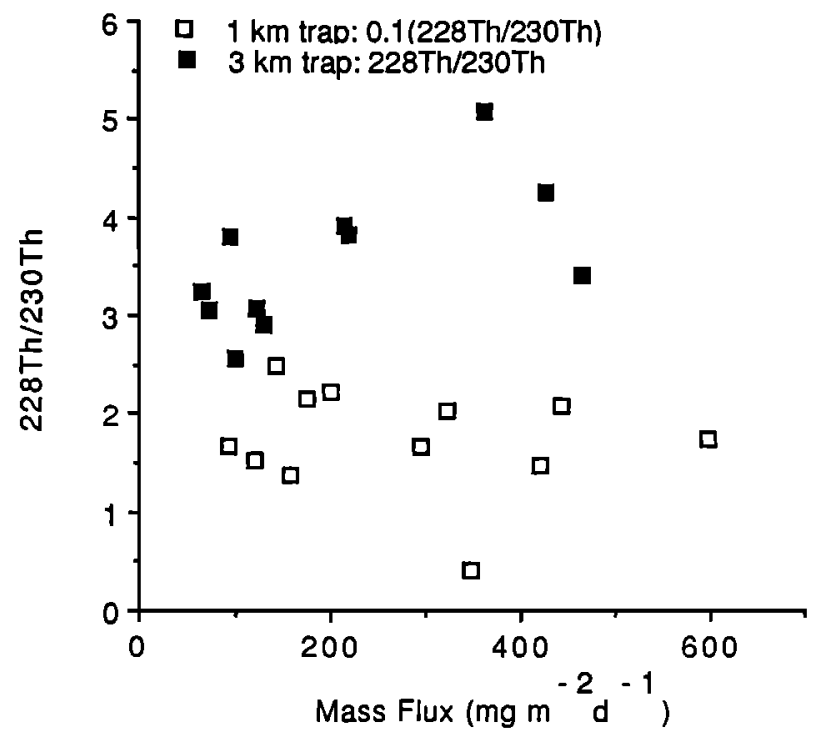

b

Fig. 3. (a) Station PAPA mass flux data for the two week sampling intervals at the two sediment trap depths. Note the approximately two week offset in mass flux changes between the traps. This implies a sinking rate of order $200 \mathrm{~m} \mathrm{~d}^{-1}$. (b) ${ }^{228} \mathrm{Th} /{ }^{230} \mathrm{Th}$ activity ratios for sediment trap material versus mass flux. The interval 11 sample from the $3800 \mathrm{~m}$ trap is omitted from this plot. The ratio tends to increase with mass flux in material at $3800 \mathrm{~m}$ whereas there is an almost constant ratio at $1000 \mathrm{~m}$. Note that the ${ }^{228} \mathrm{Th} /{ }^{230} \mathrm{Th}$ ratios for the $1000 \mathrm{~m}$ trap are multiplied by 0.1 .

${ }^{228} \mathrm{Th} /{ }^{230} \mathrm{Th}$ ratios in the large and small particle phases should be nearly equal. In the Station $\mathrm{P}$ data the large and small particle ${ }^{228} \mathrm{Th} /{ }^{230} \mathrm{Th}$ ratios differ (Table 4). This implies that $\beta_{2}$ and $\beta_{-2}$ are smaller than the ${ }^{228} \mathrm{Th}$ decay constant and/or smaller than the rate large particles travel through the water column. Two important assumptions used by Nozaki et al. [1987], which strongly affect their solutions for $\beta_{2}$ and $\beta_{-2}$, are that total and particulate surface ${ }^{230} \mathrm{Th}$ activities can be found by linear regression.
The validity of this assumption is questionable considering the particle concentration dependence of $k_{1}$ and the likely variation of $\beta_{2}$ and $\beta_{-2}$ with depth. They assume that $k_{3}$ (equivalent to our $\beta_{2}$ ) equals $P / a$ and that $k_{4}$ (equivalent to our $\beta_{-2}$ ) equals $(A S-P) / a$, where $P$ is the production rate of ${ }^{230} \mathrm{Th}, a$ is the intercept of the particulate ${ }^{230} \mathrm{Th}$ activity, $A$ is the slope of the particulate ${ }^{230} \mathrm{Th}$ activity versus depth, and $S$ is the large particle settling velocity. Using the Station $\mathrm{P}$ data and the technique given in Nozaki et al. [1987] we find a value of $0.6 \mathrm{y}^{-1}$ for $\beta_{2}$ and 62.5 $\mathrm{y}^{-1}$ for $\beta_{-2}$ (assuming a settling velocity of $100 \mathrm{~m} \mathrm{~d}^{-1}$ ). These values are closer to the results of Nozaki et al. [1987] than the values predicted by the inversion technique. The difference is primarily due to the larger intercept found using our particulate ${ }^{230} \mathrm{Th}$ data. We should note that the intercepts found with our data and with Nozaki et al. [1987] are not statistically different from zero.

The rate constants for Station $P$ predicted with the inverse technique are less sensitive to a $10 \%$ change in the activity of a given phase than the rate constants predicted from the finite difference model data. The sensitivity tests show that $k_{1}$ and $k_{-1}$ are most sensitive to changes in ${ }^{234} \mathrm{Th}$ dissolved activity and that $\beta_{2}$ and $\beta_{-2}$ are most sensitive to changes in ${ }^{228} \mathrm{Th}$ small particle activity. These results are similar to those found with the finite difference model data; however, the magnitude of the changes are smaller. For example, increasing the ${ }^{228} \mathrm{Th}$ small particle activity by 10\%: decreased $P_{L}$ to $7 \times 10^{-2} \mu \mathrm{g} \mathrm{L}^{-1}$, increased $\beta_{-2}$ to 250 $\mathrm{y}^{-1}$, and increased $\beta_{2}$ to $0.25 \mathrm{y}^{-1}$. The reduced sensitivity of the rate constants predicted with the Station $\mathrm{P}$ data may be related to the fact that the columns in the data matrix are not as collinear as those in the data matrix from the finite difference model. The different ${ }^{228} \mathrm{Th} /{ }^{230} \mathrm{Th}$ ratios of the small and large particle phases reduce the collinearity. The different ratios also tend to force the rate constants predicted by the inverse technique towards smaller values.

Including the equation for the change in large particle mass with time (Equation (2c)) in the inversion would provide additional information that could further constrain the solution for $\beta_{2}$ and $\beta_{-2}$. We need the concentration of suspended matter if we are to include the large particle equation. Unfortunately, we do not have suspended particle concentrations for Station P.

The rate constants predicted by the inverse technique using Station $\mathrm{P}$ data appear reasonable, especially when one considers the variability in mass flux (Figure 3 ). The uncertainty for each term predicted by the inverse technique ranges from 5 to $25 \%$ of the predicted value. The high large particle concentration seems unreasonable. The model assumes this concentration represents the time weighted average of material collected by the sediment traps and that there is a sharp distinction between the large particles settling at a single, constant velocity and the non-sinking, suspended small particles. These assumptions, of course, are not completely correct. The validity of the predicted $P_{L}$ and the model assumptions can be tested by examining predictions based on $P_{L}$.

Two parameters can be predicted with the large particle concentration: the small particle concentration and the large particle settling velocity. Using the particle model from Figure $1 b$ and the steady state equation for the large particles from Equation (2c), one can solve for a small particle concentration $\left(P_{s}\right)$ : 
TABLE 5. Rate Constants and Large Particle Concentration Predicted Using Station PAPA Data

\begin{tabular}{cccc}
\hline Unknown & Station P & Western Pacific & Panama Basin \\
\hline$k_{1}$ & $0.47 \pm 0.09 \mathrm{y}^{-1}$ & $0.19-0.44 \mathrm{y}^{-1}$ & $0.204-1.28 \mathrm{y}^{-1}$ \\
$k_{-1}^{\prime}$ & $0.97 \pm 0.09 \mathrm{y}^{-1}$ & $0.88-1.88 \mathrm{y}^{-1}$ & $1.33-6.3 \mathrm{y}^{-1}$ \\
$\beta_{2}$ & $0.22 \pm 0.01 \mathrm{y}^{-1}$ & $2.36-12.25 \mathrm{y}^{-1}$ & \\
$\beta_{-2} P_{L}$ & $10 \pm 1 \mu \mathrm{L}^{-1} \mathrm{y}^{-1}$ & & \\
$P_{L}$ & $12.2 \pm 2.3 \mu \mathrm{L} \mathrm{L}^{-1}$ & & \\
$\beta_{-2}$ & $0.8 \pm 0.2 \mathrm{y}^{-1}$ & $148-785^{*} \mathrm{y}^{-1}$ & \\
\hline
\end{tabular}

Errors are one standard deviation. The relatively large error in $\beta_{-2}$ is due to the inclusion of covariance between $P_{L}$ and $\beta_{-2} P_{L}$ in the error calculations. Values for the Western Pacific are from Nozaki et al. [1987], values from the Panama Basin are from Bacon and Anderson [1982]. Numbers in these two columns give the range of rate constants calculated from different profiles and at different depths. The $k_{1}$ in Bacon and Anderson is correlated with particle concentration.

* Assuming a $100 \mathrm{~m} \mathrm{~d}^{-1}$ settling velocity for the large particles.

$$
P_{a}=\frac{P_{L} \beta_{-2}+\omega \partial P_{L} / \partial z}{\beta_{2}}
$$

This equation and data in Tables 4 and 5 yield a low small particle concentration of $0.54 \mu \mathrm{g} \mathrm{L}^{-1}$. The small particle concentration predicted in this manner is very sensitive to changes in $P_{L} \beta_{-2}$ or $\omega \partial P_{L} / \partial z$, but this calculation shows that $P_{s}$ is inconsistent with expected small particle concentrations, which are probably near $10 \mu \mathrm{g} \mathrm{L}^{-1}$.

The settling velocity, $\omega$, of large particles at Station $P$ can be found from the $P_{L}$ predicted with the inverse technique (Table 5) and the average of the average mass flux at each depth (Table 4), $\bar{F}_{P_{L}}$ :

$$
\omega=\bar{F}_{P_{L}} / P_{L}
$$

Using this equation we find $\omega=20 \mathrm{~m} \mathrm{~d}^{-1}$, a relatively slow settling velocity. There are a wide range of reported settling velocities for material collected by sediment traps. Fecal pellet settling velocities tend to be on the order of 100-200 $\mathrm{m} \mathrm{d}^{-1}$. However, they tend to represent only a small fraction of the mass flux collected by sediment traps [Pilskaln and Honjo, 1987]. Estimates of marine snow settling velocities tend to be lower. Kajihara [1971] measured settling velocities of marine particles that averaged around $70 \mathrm{~m}$ $\mathrm{d}^{-1}$ but ranged from 20 to $250 \mathrm{~m} \mathrm{~d}^{-1}$. This material was collected in the field, disaggregated during sampling and transport, returned to the lab, allowed to "re-aggregate" in the laboratory, and collected with a pipette before the settling velocity was measured. Asper [1987] calculated settling velocities of trapped material that ranged from 1 to $36 \mathrm{~m} \mathrm{~d}^{-1}$ based on photographs of the material and estimates of particle concentrations. Using theoretical considerations, Hawley [1982] calculated settling velocities for particles smaller than $50 \mu \mathrm{m}$ that were less than $50 \mathrm{~m}$ $\mathrm{d}^{-1}$. Particles in this size range can provide the largest fraction of the measured mass flux [Honjo, 1978; Pilskaln and Honjo, 1987], though they may be produced by the breakup of larger particles [Honjo et al., 1982]. Thus, it appears that our predicted $\omega$ is on the low end of the observed range of settling velocities.

We can also estimate a settling velocity from the offset of mass flux peaks versus time [Honjo, 1984; Takahashi, 1986]. The peak offset (Figure 3a), though not distinct, suggests a settling velocity on the order of $200 \mathrm{~m} \mathrm{~d}^{-1}$, which disagrees by a factor of 10 with our $\omega$ calculated from the results of the inverse technique.

A possible cause of the inconsistent predictions based on the large particle concentrations may be incorrect model assumptions. There may be more than one class of large particles. Wakeham and Canuel [1988] argue that organic geochemical data is consistent with two classes of large particles, each with settling velocities on the order of 100 $\mathrm{m} \mathrm{d}^{-1}$. Mass flux variations could be associated with changes in the relative abundance of fecal pellets and marine snow and alter the composition and settling velocity of our average large particle. Rapidly sinking fecal pellets produced during a bloom in the surface water could traverse the water column at a different rate than marine snow and cause the ratio of fecal pellets to marine snow to change with time. This problem would be minimized with sufficiently long sediment trap records.

The isotopic signal of two particle classes should differ if they originate at different depths in the water column or if they have different interactions with the dissolved and small particle phases. The ${ }^{228} \mathrm{Th} /{ }^{230} \mathrm{Th}$ sediment trap data suggest that more than one class of sinking particles exist or that particle interactions change with mass flux (Figure $3 \mathrm{~b}$, note that the $1 \mathrm{~km}$ trap values are divided by 10 ). The ${ }^{228} \mathrm{Th} /{ }^{230} \mathrm{Th}$ ratio of trapped material at $3800 \mathrm{~m}$ tends to increase slightly with mass flux, while in the $1 \mathrm{~km}$ trap, the ratio remains relatively constant with increasing mass flux. The increase in ${ }^{228} \mathrm{Th} /{ }^{230} \mathrm{Th}$ with mass flux in the deeper trap suggests that more material is coming directly from the surface during times of high mass flux.

The average ${ }^{228}$ Th flux declines by $\approx 20 \%$ and the average mass flux declines by $\approx 25 \%$ between $1000 \mathrm{~m}$ and 3800 $m$ (Table 4). This relatively small decrease in the ${ }^{228} \mathrm{Th}$ activity flux implies that the large particles that carry ${ }^{228} \mathrm{Th}$ from the surface exchange ${ }^{228} \mathrm{Th}$ slowly. The slow exchange of thorium is inconsistent with increasing dissolved ${ }^{230} \mathrm{Th}$ activity with depth [Bacon and Anderson, 1982].

There are other possible reasons for the unrealistically high large particle concentrations such as deviations from steady state conditions and effects of lateral advection. These factors could be minimized by using data averaged over longer time intervals of one or more years, and by sampling at appropriate locales. 


\section{Possible Model Adjustments}

The high $P_{L}$ concentration obtained by the inversion of the Station $\mathrm{P}$ data, the low $\omega$ and $P_{s}$ predicted with $P_{L}$, the changing thorium ratios with mass flux (Figure $3 \mathrm{~b}$ ), and the small drop in the ${ }^{228} \mathrm{Th}$ flux between the two traps imply that modifications of the existing model could better describe the processes occurring in the water column. We briefly mention three possible changes in the model: 1) an additional class of sinking particles, 2) direct adsorption and desorption between the large particles and the dissolved phase, and 3) incorporation of the fact that there is a continuous range of particle sizes and settling velocities. A possible way to explain these observations would be to include two sinking particle classes. One type might represent fecal pellets that slowly interact with suspended matter. The other might represent amorphous marine snow aggregates that extensively interact with suspended particles by breaking up and re-forming frequently. Each type of particle would be characterized by different values for $\beta_{2}$ and $\beta_{-2}$ and the relative activity flux of each particle class could vary with changes in total mass flux. It may be that at depth the ${ }^{228}$ Th cycle is influenced more by the first type of large particle while the ${ }^{234} \mathrm{Th}$ and ${ }^{230} \mathrm{Th}$ cycles are influenced more by the second.

Direct interaction between the large particle and dissolved phases may also be important. If $\beta_{2}$ and $\beta_{-2}$ can be as small as, or smaller than, that predicted with the Station $P$ data, then there could be a significant amount of inorganic desorption from large particles (and adsorption onto large particles, depending on their concentration and surface characteristics).

Finally, a complete description of particle dynamics would include the continuous nature of particle size and settling velocity [McCave, 1975, 1984; Brun-Cottan, 1986]. The material collected with large volume filtration and with sediment traps does not collect sharply defined size classes and sinking velocities. Filtration yields a mass weighted particle class and sediment traps sample a flux weighted particle class.

We recognize that these modifications may not adequately represent processes occurring in the water column. We wish to emphasize, however, that one can use the procedure outlined in this paper, along with additional field data, to predict rate constants for altered (or new) models and to test model predictions.

We also note that the constant betas used in the current model are inconsistent with sediment trap observations by Martin et al. [1987]. An analytical solution to Equations (2b) and (2c), assuming that $\omega$ and the $\beta$ 's are constant and that the $T_{(\text {) }}$ 's are negligible, is

$$
\begin{aligned}
P_{L} & =P_{L_{0}} \exp \left(-z / z_{*}\right) \\
z_{*} & =\frac{\omega\left(\beta_{-1}+\beta_{2}\right)}{\beta_{-1} \beta_{-2}} \\
P_{s} & =\frac{\beta_{-2}}{\beta_{-1}+\beta_{2}} P_{L}
\end{aligned}
$$

where $P_{L_{0}}$ is the concentration of large particles at $z=0$. The exponential function for the large particle distribution contradicts the power law relationship for mass flux found by Martin et al. [1987], and gives negligible particle concentrations at depth. This suggests that the model will fit oceanic observations only if we permit the $\beta$ 's to vary with depth. More realistic models of the physical and biological particle cycling processes, such as those explored by Toggweiler et al. [1987] and Sarmiento et al. [1989], should include more fundamental rate constants that apply over larger oceanic regions.

\section{Conclusions}

Models of trace metal and particle behavior in the ocean show basic similarities. As a result, thorium isotope data can provide information on particle cycling rate constants as well as thorium adsorption and desorption rate constants. These rate constants can be found through the solution of a system of equations that describe the distribution of mass and activity between the various phases. An important feature of these models is that the data for the system of equations can be collected with sediment traps and samples of dissolved and suspended material.

We used a finite difference model to generate predicted profiles of thorium isotopes in solution, in suspended material, and in sinking particles. These data were used to test the inverse technique. We tested the sensitivity of the rate constants predicted with the inverse technique by changing the activity of different phases. The rate constants most sensitive to changes in the data are $\beta_{2}$ and $\beta_{-2}$. This sensitivity is associated with the model rate constants being larger than any of the thorium isotope decay constants.

Rate constants and the concentration of large particles were predicted from data collected at Station P. Values of $k_{1}\left(0.47 \pm 0.09 \mathrm{y}^{-1}\right)$ and $k_{-1}\left(0.97 \pm 0.09 \mathrm{y}^{-1}\right)$ are within the range of previous estimates. $\beta_{2}\left(0.22 \pm 0.01 \mathrm{y}^{-1}\right)$ and $\beta_{-2}\left(0.8 \pm 0.2 \mathrm{y}^{-1}\right)$ are much smaller than other estimates [Nozaki et al., 1987] made with thorium isotope data. The difference could be due to the assumptions used by Nozaki et al. [1987], to non-steady state conditions at Station $P$, or to multidimensional effects at Station P. The predicted concentration of large particles is very high, on the order expected for the suspended particle concentration.

Analysis of the Station $\mathrm{P}$ data imply that modifications of the current particle model may be needed. Possible model modifications include: an additional class of sinking particles, direct interaction between the large particle and dissolved phases, and explicitly including the continuous nature of particle size and settling velocity. The following measurements should be obtained in order to test such models:

1. In sediment traps, the mass flux of particles and the activity per unit mass of the three thorium isotopes, ${ }^{228} \mathrm{Th}$, ${ }^{230} \mathrm{Th}$, and ${ }^{234} \mathrm{Th}$. These measurements need to be obtained at a minimum of two depths spanning the portion of the water column that is of interest. Ideally, more traps, spaced as closely as possible, should be used.

2. In the water column, the concentration of dissolved nutrients and of the three thorium isotopes. These measurements should be obtained between the sediment traps, and at the depth of the traps, with adequate resolution to define the average between the traps.

3. Also in the water column, the concentration of suspended particles and the activity per unit mass of the three thorium isotopes on the particles. Again, there should be measurements at the depth of the traps and between 
the traps, with sufficient resolution to define the average between the traps.

4. The dissolved activity of ${ }^{228} \mathrm{Ra}$. ${ }^{228} \mathrm{Ra}$ activities are highly variable and need to be measured at all locations with adequate vertical resolution to resolve the average between sediment traps.

The nutrient and small particle concentration measurements let one include Equations (2a) and (2b), and possibly determine the importance of $\beta_{-1}$.

In concluding, we note that physical oceanographers often attempt to determine with conservative tracers the transport terms lumped into our parameter $T$. Such studies have recently begun to include non-conservative tracers, such as nutrients [e.g., Rintoul, 1988; and Schlitzer, 1988], in which the mainly biologically mediated source and sink terms are included in a single unknown directly analogous to our term $T$. In this paper we expand the source and sink terms in a model of particle and thorium cycling that is based on earlier trace metal cycling studies, and we outline how to use observations to constrain these terms. Future work will eventually bring together techniques that emphasize the physical processes with those that emphasize the chemical and biological and will require physicists, chemists and biologists to obtain data sets with better spatial and temporal resolution. The Global Ocean Flux Study and the World Ocean Circulation Experiment promise to do this for us.

Acknowledgments. Work by R.J.M. and J.L.S. was supported by ONR contract N00014-86-K-0063.P2 and NSF-GOFS grant OCE-8711905. M.P.B. acknowledges support form NSF grant OCE-8800620 and ONR contract N00014-87-K-0007. Sediment trap samples were provided by S. Honjo, and technical assistance was given A. P. Fleer and T. R. Hammer. R. Toggweiler, S. Rintoul, J. H. Martin, J. W. Murray, and an anonymous reviewer helped to improve the manuscript.

\section{REFERENCES}

Asper, V. L., Measuring the flux and sinking speed of marine snow aggregates, Deep-Sea Res., 34, 1-17, 1987.

Bacon, M. P., and R. F. Anderson, Distribution of thorium isotopes between dissolved and particulate forms in the deep sea, J. Geophys. Res., 87, 2045-2056, 1982.

Bacon, M. P., C.-A. Huh, A. P. Fleer, and W. G. Deuser, Seasonality in the flux of natural radionuclides and plutonium in the deep Sargasso Sea, Deep-Sea Res., 32, 273-286, 1985.

Betzer, P. R., W. J. Showers, E. A. Laws, C. D. Winn, G. R. DiTullio, and P. M. Kroopnick, Primary productivity and particle fluxes on a transect of the equator at $153^{\circ} \mathrm{W}$ in the Pacific Ocean, Deep-Sea Res., 31, 1-11, 1984.

Bishop, J. K. B., D. R. Ketten, and J. M. Edmond, The chemistry, biology and vertical flux of particulate matter from the upper $400 \mathrm{~m}$ of the Cape Basin in the southeast Atlantic Ocean, DeepSea Res., 25, 1121-1161, 1978.

Brun-Cottan, J. C., Vertical transport of particles within the ocean, in The Role of Air-Sea Exchange in Geochemical Cycling, edited by P. Buat-Menard, pp. 83-111, D. Reidel, Hingham, Mass., 1986.

Clegg, S., and J. L. Sarmiento, The hydrolytic scavenging of metal ions by marine particulate matter, Prog. Oceanog., 23, 1-21, 1989.

Coale, K. H., and K. W. Bruland, ${ }^{234} \mathrm{Th}^{238} \mathrm{U}$ disequilibria within the California Current, Limnol. Oceanogr., 30, 22-33, 1985.

Coale, K. H., and K. W. Bruland, Oceanic stratified euphotic zone as elucidated by ${ }^{234} \mathrm{Th}:{ }^{238} \mathrm{U}$ disequilibria, Limnol. Oceanogr., 32, 189-200, 1987.
Cochran, J. K., The geochemistry of ${ }^{226} \mathrm{Ra}$ and ${ }^{228} \mathrm{Ra}$ in marine deposits, Ph.D. dissertation, 260 pp., Yale Univ., New Haven, Conn., 1979.

Collier, R., and J. Edmond, The trace element geochemistry of marine biogenic particulate matter, Prog. Oceanogr., 13, 113-199, 1984.

Fisher, N. S., On the reactivity of metals for marine phytoplankton, Limnol. Oceanogr., 31, 443-449, 1986.

Fisher, N. S., P. Bjerregaard, and S. W. Fowler, Interactions of marine plankton with transuranic elements. 1. biokinetics of neptunium, plutonium, americium, and californium in phytoplankton, Limnol. Oceanogr., 28, 432-447, 1983.

Hawley, N., Settling velocity distribution of natural aggregates, J. Geophys. Res., 87, 9489-9498, 1982.

Honjo, S., Sedimentation of materials in the Sargasso Sea at a 5,367 m deep station, J. Mar. Res., 36, 469-492, 1978.

Honjo, S., Material fluxes and modes of sedimentation in the mesopelagic and bathypelagic zones, J. Mar. Res., 38, 53-97, 1980.

Honjo, S., Study of ocean fluxes in time and space by bottorntethered sediment trap arrays: A recommendation, in Global Ocean Flux Study, Proceedings of a Workshop, pp. 306-324, National Academy Press, Washington, D. C., 1984.

Honjo, S., S. J. Manganini, and J. J. Cole, Sedimentation of biogenic matter in the deep ocean, Deep-Sea Res., 29, 609-625, 1982.

Honeyman, B. D., L. S. Balistrieri, and J. W. Murray, Oceanic trace metal scavenging: the importance of particle concentration, Deep-Sea Res., 35, 227-246, 1988.

Kajihara, M., Settling velocity and porosity of large suspended particles, J. Oceanogr. Soc. Japan, 27, 158-162, 1971.

Kumar, M. D., Cation hydrolysis and the regulation of trace metal composition in seawater, Geochim. Cosmochim. Acta, $51,2137-2145,1987$.

Li, Y.-H., Ultimate removal mechanisms of elements from the ocean, Geochim. Cosmochim. Acta, 45, 1659-1664, 1981.

McCave, I. N., Vertical flux of particles in the ocean, Deep-Sea Res., 22, 491-502, 1975.

McCave, I. N., Size spectra and aggregation of suspended particles in the deep ocean, Deep-Sea Res., 31, 329-352, 1984.

Martin, J. H., G. A. Knauer, D. M. Karl, and W. W. Broenkow, VERTEX: carbon cycling in the northeast Pacific, Deep-Sea Res., 34, 267-285, 1987.

Nozaki, Y., Y. Horibe, and H. Tsubota, The water column distributions of thorium isotopes in the western North Pacific, Earth Planet. Sci. Lett., 54, 203-216, 1981.

Nozaki, Y., H.-S. Yang, and M. Yamada, Scavenging of thorium in the ocean, J. Geophys. Res., 92, 772-778, 1987.

Pilskaln, C. H., and S. Honjo, The fecal pellet fraction of biogeochemical particle fluxes to the deep sea, Global Biogeochem. Cycles, 1, 31-48, 1987.

Rintoul, S. R., Mass, heat and nutrient fluxes in the Atlantic Ocean determined by inverse methods, Ph.D. dissertation, 287 pp., MIT/WHOI, Woods Hole, Mass., 1988.

Sarmiento, J. L., A study of mixing in the deep sea based on STD, radon-222, and radium-228 measurements, $P h . D$. dissertation, 283 pp., Columbia Univ., New York, N.Y., 1978.

Sarmiento, J. L., M. J. R. Fasham, U. Siegenthaler, R. Najjar, and J. R. Toggweiler, Models of chemical cycling in the oceans: Progress report II, Tech. Rep. \#6, p.46, Ocean Tracers Laboratory, Princeton Univ., Princeton, N.J., 1989.

Schlitzer, R., Modeling the nutrient and carbon cycles of the North Atlantic 1. Circulation, mixing coefficients, and heat fluxes, J. Geophys. Res., 93, 10,699-10,723, 1988.

Suess, E., Particulate organic carbon flux in the oceans-surface productivity and oxygen utilization, Nature, 288, 260-263, 1980.

Takahashi, K., Seasonal fluxes of pelagic diatoms in the subarctic Pacific 1982-1983, Deep-Sea Res., 33, 1225-1251, 1986.

Toggweiler, J. R., J. L. Sarmiento, R. Najjar, and D. Papademetriou, Models of chemical cycling in the oceans: A progress report, Tech. Rep. \#4, p.61, Ocean Tracers Laboratory, Princeton Univ., Princeton, N.J., 1987.

Turner, D. R., M. Whitfield, and A. G. Dickson, The equilibrium speciation of dissolved components in freshwater and seawater 
at $25^{\circ} \mathrm{C}$ and $1 \mathrm{~atm}$ pressure, Geochim. Cosmochim. Acta, 45 , 855-881, 1981.

Wakeham, S. G., and E. A. Canuel, Organic geochemistry of particulate matter in the eastern tropical North Pacific Ocean: Implications for particle dynamics, J. Mar. Res., 46, 183-213, 1988.

Walsh, I., J. Dymond, and R. Collier, Rates of recycling of biogenic components of settling particles in the ocean derived from sediment trap experiments, Deep-Sea Res., 35, 43-58, 1988.

Wolberg, J. R., Prediction Analysis, 291 pp., D. Van Nostrand, Princeton, N.J., 1967.
M. P. Bacon, Department of Chemistry, Woods Hole Oceanographic Institution, Woods Hole, MA 02543.

R. J. Murnane and J. L. Sarmiento, Program in Atmospheric and Oceanic Sciences, Princeton University, Princeton, NJ 08544.
(Received May 23, 1989; revised January 5, 1990; accepted March 20, 1990.) 